\section{Response to Baertling et al.}

To the Editor:

Baertling and colleagues report an 18-month-old previous early preterm child with biochemical signs of carbonic anhydrase VA (CAVA) deficiency, later genetically confirmed, and a fatal outcome. ${ }^{1}$ In the patient, they report concurrent arterial hypertension. The patient received early and adequate therapeutic measures, which led to rapid normalization of the biochemical abnormalities. ${ }^{2}$ Despite this, the patient died after developing severe intracranial hypertension, brain edema, and herniation.

The authors conclude that "this disease can be fulminant and fatal despite ... adequate treatment." ${ }^{\prime \prime}$ While this comment is certainly entirely correct, it is not so clear what caused the fatal course in this patient. According to the information provided, the metabolic decompensation due to CAVA deficiency was fully resolved after 24 hours. The following clinical course may therefore be entirely caused by the arterial hypertension that was present at manifestation without any obvious explanation. It is our view that in fact the uncontrolled arterial hypertension is more likely the cause of the fatal course than sequelae from metabolic decompensation. Levels of ammonia, blood gases, and lactate as reported were still in a range allowing for complete correction after the fully adequate treatment that the patient received. Since encephalopathy persisted despite this normalization, the possibility of causation due to arterial hypertension should be considered.
It would have been good though to know in more detail about the maximum levels of ammonia and lactate, and whether other biochemical hallmarks of CAVA deficiency were present (such as hypoglycemia or ketosis). As well, exact magnitude of arterial hypertension would have been interesting for a full understanding. Finally, regarding the identified homozygous deletion of the first two exons of the CA5A gene, this variant clearly destroys the normal gene function. ${ }^{2}$ Absence of metabolic decompensation in this patient after birth could be the result of the early anabolic treatment that he likely received when born preterm at 26 weeks.

\section{DISCLOSURE}

The authors declare no conflicts of interest.

Publisher's note Springer Nature remains neutral with regard to jurisdictional claims in published maps and institutional affiliations.

\section{Johannes Häberle, $M D$ (D) $^{1}$ and Véronique Rüfenacht, $M S c^{1}$}

${ }^{1}$ Division of Metabolism, University Children's Hospital Zurich, Zurich, Switzerland. Correspondence: Johannes Häberle (Johannes.haeberle@kispi.uzh.ch)

\section{REFERENCE}

1. Baertling $F$, et al. Fatal metabolic decompensation in carbonic anhydrase VA deficiency despite early treatment and control of hyperammonemia. Genet Med. 2019; https://doi.org/10.1038/s41436-019-0677-9.

2. Diez-Fernandez $C$, et al. Defective hepatic bicarbonate production due to carbonic anhydrase VA deficiency leads to early-onset life-threatening metabolic crisis. Genet Med. 2016;18:991-1000.

Advance online publication 22 October 2019. doi:10.1038/s41436-019-0678-8 